\title{
Inventaire et évolution des flores fongiques de surface du reblochon de Savoie
}

\author{
C Bärtschi, J Berthier, G Valla
}

Université Claude-Bernard Lyon I, Laboratoire de mycologie : biosystématique et nuisances fongiques, bâtiment 405, 43, bd du 11 Novembre 1918, 69622 Villeurbanne Cedex, France

(Reçu le 18 octobre 1993; accepté le 11 janvier 1994)

Résumé - Une étude quantitative au cours de l'affinage des flores fongiques de surface de reblochons fermiers et fruitiers est réalisée avec la méthode des ufc. De ce point de vue, l'évolution des populations levures et moisissure(s) est la même pour toutes les fabrications. Sur le reblochon, la croissance de Geotrichum candidum, seule moisissure de ce fromage, et la multiplication des levures se font essentiellement entre $J+1$ et $J+8$. D'un point de vue qualitatif, 13 espèces de levures dominantes ont été identifiées avec les systèmes API ATB $32 \mathrm{C}$ et API ANAER combinés et amendés. De ces 13 espèces, Candida famata est la seule qui soit dominante sur tous les fromages fruitiers ou fermiers. Candida vini est caractéristique des reblochons fermiers. En revanche, Candida zeylanoides est caractéristique des productions fruitières qui, par ailleurs, sont plus pauvres en espèces de levures dominantes que les productions fermières.

fromage / affinage / Geotrichum / levures / diagnose / Api system

Summary - Evolution of the surface fungal flora of Reblochon cheese. The Reblochon of Savoie is a pressed uncooked French cheese with a washed rind. The cheeses are made with unpasteurized bovine milk. This work is a qualitative and quantitative study of the fungal population on the surface of ripening farm and dairy cheeses. Geotrichum candidum was the only mold of the Reblochon; it developed essentially on the surface from day +1 to day +8 . Thirteen main predominant yeasts have been identified with the computer program of Barnett et al (1990b). Candida famata emerged as the most prevalent yeast of the Reblochon rind. It was dominant on the surface of all the farm and dairy cheeses tested. Candida vini has been isolated only from farm cheeses. On the contrary, Candida zeylanoides characterized the dairy cheese. On the cheese surface, the number of yeasts increased rapidly and essentially from day +1 to day +8 . A similar increase was seen for $\mathrm{G}$ candidum. From day +8 to day +36 the fungal population of the rind stabilized.

cheese / ripening / Geotrichum / yeast / diagnosis / Api system 


\section{INTRODUCTION}

À notre connaissance, la microflore du reblochon n'a jamais fait l'objet d'une étude approfondie. De plus, il est difficile, sinon impossible, d'extrapoler les résultats obtenus sur d'autres fromages de type croûte fleurie comme le camembert (Schmidt et Lenoir, 1978, 1980; Schmidt et Daudin, 1983) ou les fromages de chèvre (Nahabieh et Schmidt, 1990) ou même les fromages de type croûte lavée tels que les munster, livarot, maroilles. En effet, la croûte du reblochon est intermédiaire entre celle de ces fromages.

Ce travail est une étude comparée de reblochons fermiers et fruitiers. II a pour objectifs l'inventaire et l'évolution qualitatifs et quantitatifs de la microflore fongique de surface du reblochon au cours de l'affinage.

Notre étude s'insère dans le cadre d'un programme plus général, commandité par le Syndicat interprofessionnel du reblochon, où l'Institut technique du gruyère (La Rochesur-Foron, 74) s'est chargé de l'inventaire des flores bactériennes ainsi que du suivi des caractères physico-chimiques (Chamba et Perron, 1991).

\section{MATÉRIEL ET MÉTHODES}

\section{Origine des échantillons}

Les reblochons proviennent de 5 fabrications différentes (A, B, C, D, E) considérées par les professionnels comme représentatives de produits fermiers ( $A$ et $B$ ) et fruitiers $(C, D$ et $E)$ de qualité.

$\mathrm{J}$ étant le jour du caillage du lait, 4 stades d'affinage ont été retenus pour cette étude : $\mathrm{J}+$ 1 correspond à des fromages après saumurage, sans croûte distincte; $J+8$ correspond à des fromages fermiers avant lavage et à des fromages fruitiers ayant subi les premiers soins, soit un trempage dans une suspension aqueuse de Geotrichum candidum pour la fabrication fruitière $\mathrm{C}$, soit 3 frottages avec une suspension de $G$ candidum dans l'eau salée pour les fabrications fruitières $D$ et $E ; J+15$ correspond à des fromages à l'emballage, affinés $8 \mathrm{j}$ à $12-14^{\circ} \mathrm{C}$ $(\mathrm{A}, \mathrm{C}, \mathrm{D}, \mathrm{E})$ ou à $16^{\circ} \mathrm{C}(\mathrm{B})$; enfin, $\mathrm{J}+36$ correspond à des fromages conservés 3 semaines sous emballage à des températures de l'ordre de 5 à $10^{\circ} \mathrm{C}$.

\section{Prélèvements et traitements des échantillons}

Les prélèvements pour l'étude microbiologique sont effectués en surface des fromages sous forme de "scalps" (= lamelles) de 1 à $2 \mathrm{~mm}$ d'épaisseur, découpés à l'aide d'un épluchelégumes stérile.

Pour chaque stade, $25 \mathrm{~g}$ de "scalps" sont obtenus à partir de 4 fromages de même origine.

L'échantillon est broyé et homogénéisé à l'aide d'un broyeur ultra-turrax pendant $30 \mathrm{~s}$ (20 000 tours $/ \mathrm{min}$ ) dans un diluant stérile de composition (g/l) : peptone de caséine : 20,0; chlorure de sodium : 8,5; tween $20: 30 \mathrm{ml}$ - pH 6,6. Le broyat est introduit dans une fiole jaugée stérile et complétée à $250 \mathrm{ml}$ (dilution $10^{-1}$ de fromage) avec le même diluant, des dilutions successives de raison 10 sont ensuite réalisées avec le diluant jusqu'à $10^{-11}$.

\section{Dénombrements des micro-organismes}

La technique appliquée est celle des unités formant colonies (ufc) plus particulièrement adaptée au dénombrement des micro-organismes unicellulaires, donc aux levures.

Malheureusement, il n'y a pas encore actuellement de méthodes permettant de dénombrer ou de quantifier précisément dans les milieux naturels les moisissures, micro-organismes filamenteux pluricellulaires.

Cependant, la méthode des ufc, si elle est rigoureusement standardisée d'un échantillon à l'autre, en particulier pour les broyages, permet de mettre en évidence les moisissures dominantes et d'apprécier leur développement, c'est-à-dire leur croissance. 
La méthode des ufc, lorsque c'est possible comme pour cette étude, doit être associée à une observation préalable des échantillons sous le microscope stéréoscopique, afin d'y déceler les moisissures contaminantes accidentelles.

À partir de chaque dilution de l'échantillon broyé et jusqu'à $10^{-11}, 2$ boîtes de Pétri (diamètre $100 \mathrm{~mm}$ ) contenant un milieu nutritif sélectif pour champignons, sont ensemencées en surface avec $0,1 \mathrm{ml}$ de suspension-dilution, étalée avec des billes de verre stériles. Le milieu nutritif MYAC a pour composition $(\mathrm{g} / \mathrm{l})$ : extrait de malt : 20,0 ; extrait de levure : 2,0 ; glucose : 2,0 : monophosphate d'ammonium : 5,0 ; chloramphénicol: 0,5 ; lait de vache écrémé : 1,0 ; gélose : $15,0-\mathrm{pH}$ 5,6 . Les boîtes ensemencées sont ensuite mises à incuber à $18^{\circ} \mathrm{C}$, à l'obscurité pendant $4 \mathrm{j}$ au moins, la détermination des moisissures nécessitant des observations ultérieures à $7-15 \mathrm{j}$. Le dénombrement des micro-organismes est réalisé sur les boîtes de Pétri présentant un nombre d'ufc compris entre 30 et 100 .

\section{Isolements des souches}

Ils sont réalisés à partir des boites gélosées nutritives comptant une centaine de colonies bien distinctes les unes des autres. Pour un reblochon et à chaque stade étudié au moins 10 isolements sont effectués aléatoirement : 9 à partir de colonies levures et 1 à partir de colonies mycéliennes. Le nombre d'isolats correspond généralement à la racine carrée du nombre d'uic pour la dilution concernée. Les levures et les moisissures sont transférées sur milieu nutritif MYAc.

Par ailleurs, toute colonie mycélienne détectable sur la croûte d'un fromage, à l'œil nu ou sous le microscope stéréoscopique, fait l'objet d'un isolement.

Pour l'ensemble des 4 stades des 5 productions étudiées, 220 isolats ont été obtenus en culture.

\section{Identifications}

\section{Moisissures}

L'identification des genres de moisissures repose, aujourd'hui encore, exclusivement sur des caractères cyto-morphologiques. Elle est réalisée avec les ouvrages de Domsch et al (1980), Ellis (1971 1976) pour les Hyphomycètes et de Sutton (1980) pour les Coelomycètes.

\section{Levures}

L'identification des levures repose essentiellement sur l'étude de leurs caractéristiques biochimiques. La méthode traditionnelle est longue et fastidieuse (Wickerham, 1951; Barnett et al, 1990a). Divers systèmes d'identification rapide sont aujourd'hui commercialisés sous forme de kit. Malheureusement, la mise en œuvre d'un seul système ne conduit pas toujours à l'identification des levures autres que celle d'intérêt médical (Ison, 1987 ; Deak et Beuchat, 1988 ; Heard et Fleet, 1990 ; Rohm et al, 1990 ; Seiler, 1991 ; Deak, 1993). La méthode utilisée pour ce travail est originale. Elle combine les systèmes API ATB $32 \mathrm{C}$ et API ANAER amendés par nos soins (tableau I).

Le système ATB $32 \mathrm{C}$ propose 30 sources de carbone à assimiler ; la lecture des tests est basée sur l'apparition d'un trouble lié à la multiplication des levures dans les cupules. Les plaques API ANAER ont permis de tester le comportement fermentaire des levures sur glucose, galactose, maltose, saccharose, lactose, raffinose, tréhalose et mélibiose. Pour l'obtention d'une anaérobiose satisfaisante, l'incubation des plaques est réalisée en jarre anaérobie sous atmosphère d' $\mathrm{H}_{2}$ et $\mathrm{CO}_{2}$. La fermentation d'un sucre se traduit dans les cupules par le virage d'un indicateur coloré de $\mathrm{pH}$. La fermentation du glucose permet entre autres la différenciation d'espèces telles que Candida famata et Candida kefyr aux métabolismes assimilatifs très proches. Les 2 systèmes API sont complétés par 2 tests biochimiques traditionnels essentiels pour la discrimination des levures autres que les Candida: l'assimilation des nitrates sur milieu YNB (Yeast Nitrogen Base $+\mathrm{KNO}_{3}$ ) et l'hydrolyse de l'urée sur milieu de Christensen.

Les tests biochimiques sont complétés par les tests de sporulation après 4 semaines de culture sur milieu de Fowell (1952) et la recherche de pseudomycélium ou de mycélium après $15 \mathrm{j}$ de culture sous lamelle sur milieu PDA (Potatoes Dextrose Agar).

Les résultats des obsenvations microscopiques et des 41 tests biochimiques sont traités avec le Barnett Computer Program (BCP) (Barnett et al, 1990b). La nomenclature utilisée ici est par conséquent celle de Barnett et al (1990a). 
Tableau I. Caractères cyto-morphologiques et biochimiques utilisés pour l'identification des souches levures.

Cyto-morphological and biochemical characters used for yeast strain identification.

1. Caractères cyto-morphologiques

- Thalle (sur milieu PDA, en “anaérobiose" : 2 semaines) :

- cellules ou pseudomycélium ou hyphes

- bourgeonnement ou cloisonnement

- Ascospores (sur milieu de Fowell : 4 semaines) :

- formation ou non d'asques

- caractères morphologiques des ascospores

2. Tests biochimiques : $\mathbf{4 1}$

Assimilation

Glucose

Galactose

Mélibiose

Levulinate

Sorbose

Lactose

Gluconate

Ribose

Raffinose

Glucuronate

Xylose

Mélézitose

Glucosamine

Arabinose

Palatinose

2-Cétogluconate

Rhamnose

-

Saccharose

Glycérol

Maltose

Erythritol

Sorbitol

Tréhalose

Mannitol

Cellobiose

Inositol

$\mathrm{N}$-Acétylglucosamine

$\alpha$-Méthylglucoside

Esculine

Lactate

-

Nitrate

Fermentation

Glucose

Lactose

Tréhalose

Galactose

Maltose

Mélibiose

Saccharose

Raffinose

Hydrolyse

Urée

Résistance

Cycloheximide

RESULTATS

\section{Étude qualitative}

\section{Moisissures}

Geotrichum candidum Link ex Leman est le seul champignon filamenteux mis en évidence sur tous les reblochons et à tous les stades. La forme imparfaite de ce champi- gnon filamenteux productrice d'arthroconidies est rangée dans les moisissures Hyphomycètes. Geotrichum candidum peut aussi être rattaché aux levures sensu lato car il présente parfois une forme parfaite avec asques non protégés dans un ascocarpe (Barnett et al, 1990a).

La comparaison des 23 isolats réalisés à partir des 5 productions révèle un mélange de 2 souches distinctes macroscopiquement en culture : une souche 
blanche, la plus fréquente et une souche blanc-grisâtre.

Soumises aux tests API $32 \mathrm{C}$ et API ANAER amendés, les 2 souches ne diffèrent qu'au niveau de l'esculine que seule la souche blanche assimile. Ces résultats confirment l'homogénéité de l'espèce (De Hoog et al, 1986).

À côté de cette espèce omniprésente, caractéristique du reblochon, un certain nombre de moisissures contaminantes et accidentelles ont été trouvées.

Mucor hiemalis Vehmer, l'une des moisissures responsable de l'accident «Poil de Chat " en fromagerie (Berthier et al, 1991) est présente dès $\mathrm{J}+8$ pour 3 des 5 productions, qu'elles soient fermières ou laitières, et à l'exception d'une production fermière, se maintient jusqu'à la fin de l'affinage.

Sur les reblochons, l'accident Mucor prend rarement l'ampleur rencontrée pour les fromages à pâte molle et à croûte fleurie, pour 2 raisons majeures. D'une part, le pressage de la pâte qui accélère l'égouttage réduit la durée de sensibilité du reblochon à une contamination par le Mucor, et d'autre part les soins périodiques du "lavage" qui éliminent ou du moins gênent le contaminant.

À noter aussi, la présence épisodique soit précoce à J + 1 de Fusarium et Phoma, soit tardive à $\mathrm{J}+36$ de Penicillium du groupe verrucosum responsable de tâches vertes indésirables.

\section{Levures}

Les 175 isolats étudiés se répartissent entre 13 espèces (tableaux II et III). Parmi cellesci, une seule Candida famata, forme anascosporogène de Debaryomyces hansenii, est présente dans toutes les productions, fermières et laitières, parfois à tous les stades de l'affinage. Candida dairensis et sa forme ascosporogène Saccharomyces dairensis ainsi que Cryptococcus laurentii sont elles aussi isolées à la fois des fromages fermiers et laitiers mais seulement dans 3 des 5 productions.

En revanche, 2 espèces n'ont été trouvées que dans l'un ou l'autre type de fabrication. Candida zeylanoides n'a été isolée que des reblochons fruitiers et à l'inverse Candida vini n'est dominante que dans les reblochons fermiers ; les 2 espèces, il faut le souligner, étant présentes à tous les stades de l'affinage.

Quatre espèces, Candida catenulata, $C$ colliculosa, $C$ polymorpha et $C$ rugosa n'ont été généralement trouvées que dans une seule production, souvent à plusieurs stades.

Enfin, 4 espèces Candida lipolytica, $C$ sphaerica forme anascosporogène de Kluyveromyces lactis, Kluyveromyces marxianus et Candida robusta et sa forme ascosporogène Saccharomyces cerevisiae ont une présence encore plus limitée puisqu'elles n'ont été isolées qu'à un stade d'une seule production.

\section{Étude quantitative}

Cette étude révèle une évolution similaire des populations de Geotrichum et de levures au cours de l'affinage, et ce tant pour les fromages laitiers que pour les fromages fermiers (tableaux IV et V).

Par ailleurs, à aucun stade il n'y a de différences très significatives dans les niveaux de populations fongiques entre les fabrications fermières et laitières.

Le développement de Geotrichum candidum se fait essentiellement lors des 8 premiers jours d'affinage. Avec cette moisissure au développement particulier associé à une fragmentation facile et spontanée des hyphes en articles courts et en arthroconidies unicellulaires, la méthode des ufcfournit des données assez significatives. 
Tableau II. Levures dominantes présentes à la surface du reblochon : répartition des isolats au cours de l'affinage ( ${ }^{*}$ forme parfaite).

Predominant yeasts on the surface of Reblochon cheese: breakdown of isolates during ripening ( ${ }^{*}$ perfect state).

Espèces (175 souches)

$J+1 \quad J+8 \quad J+15 \quad J+36$

Candida famata

$={ }^{*}$ Debaryomyces hansenii

34 souches

A

B

C

D

E

Candida zeylanoides

C

64 souches

D

E

$j+8$

"Candida dairensis"

= "Saccharomyces dairensis

13 souches

A

B

C

Cryptococcus laurentii

A

8 souches

C

D

B:2

A:2

C:2

D:3

B:1

B:2

$\mathrm{E}: 3$

D:3

E:2

D:3

D:7

C:2

C:8

E:2

E:5

D:4

D:6

C:7

C:7

E:4

E:7

D:6

D:2

$A: 1^{*}$

B:1

C: $1^{*}$

B:1

E:7

E:4

Candida vini

24 souches

A

B

C:3

C:2

B:2

B:2

C:2*

C:1*

A: 1

Candida rugosa

4 souches

A

E

D:3

D:1

Candida robusta $=$

* Saccharomyces cerevisiae

A

B

A:3

B:2

B:6

A:4

B:4

A:1

E:2

3 souches

Candida catenulata

6 souches

A

A: $1-1^{*}$

Candida polymorpha

$={ }^{*}$ Pichia triangularis

5 souches

Candida colliculosa

= " Torulaspora delbruckii

A

A:1

$A: 1$

A:1

3 souches

Candida sphaerica

$=$ " Kluyveromyces lactis

A

A:2

2 souches

Candida kefyr $=$

* Kluyveromyces marxianus

A

$A: 2^{*}$

2 souches

Candida lipolytica $=$

B

B:4

- Yarrowia lipolytica

4 souches 
Tableau III. Pourcentage d'isolements des différentes espèces de levures du reblochon en fonction du type de fabrication (175 isolats).

Percentage of the different yeast species isolated from dairy or farm Reblochon cheese (175 isolates).

Espèces

Répartition (\%)

Reblochon fruitier

Reblochon fermier

Candida catenulata

Candida colliculosa

Candida famata

Candida kefyr

Candida lipolytica

Candida polymorpha

Candida robusta

Candida rugosa

Candida sphaerica

Candida vini

Candida zeylanoides

Cryptococcus laurentii

Saccharomyces dairensis

$\begin{array}{cr}0 & 9,0 \\ 0 & 4,5 \\ \mathbf{2 7 , 5} & \mathbf{1 0 , 6} \\ 0 & 3,0 \\ 0 & 6,0 \\ 0 & 7,6 \\ 0 & 4,5 \\ 1,8 & 3,0 \\ 0 & 3,0 \\ 0 & 36,4 \\ \mathbf{5 8 , 7} & 0 \\ 6,4 & 1,5 \\ 5,5 & 10,6\end{array}$

Tableau IV. Populations de Geotrichum candidum à la surface du reblochon au cours de l'affinage. Geotrichum candidum populations on the surface of Reblochon cheese during ripening.

Productions fermières
(ufc/g)

Productions fruitières (ufc/g)
$A$
$B$
$C$
$D$
E

$\begin{array}{llllll}\mathrm{J}+1 & 1,5.10^{3} & 4,0.10^{4} & 2,2.10^{3} & 1,5.10^{3} & 3,0.10^{4} \\ \mathrm{~J}+8 & 4,0.10^{7} & 5,0.10^{7} & 7,0.10^{7} & <10^{5} & 1,0.10^{7} \\ \mathrm{~J}+15 & 2,0.10^{8} & 3,5.10^{8} & 2,4.10^{8} & 1,5.10^{7} & 5,0.10^{6} \\ \mathrm{~J}+36 & 2,2.10^{8} & 4,5.10^{8} & 1,8.10^{8} & 4,5.10^{8} & 5,5.10^{7}\end{array}$

Entre le caillé pressé et déjà égoutté et le $8^{\mathrm{e}}$ jour d'affinage la "population" du Geotrichum candidum est multipliée par $10^{3}$ ou $10^{4}$ suivant les productions. Après $\mathrm{J}$ +8 et jusqu'à la fin de l'affinage, la croissance du Geotrichum candidum à la surface des reblochons est faible (environ 1 log) ou nulle.
La population levures à $\mathrm{J}+1$ est généralement ( 4 fois sur 5 ) de l'ordre de $10^{6}$ ufc. À $\mathrm{J}+8$, la croûte du reblochon a une population de levures maximale, comprise entre $10^{8}$ et $10^{9}$ ufc selon la fabrication. Par la suite, alors que la croûte s'individualise de plus en plus, la population de levures reste stable avec $10^{8}$ ufc ou diminue un peu. 
Tableau V. Populations de levures à la surface du reblochon au cours de l'affinage. Yeast populations on the surface of Reblochon cheese during ripening.

\begin{tabular}{lccccc}
\hline Stades & $\begin{array}{c}\text { Production fermières } \\
\text { (ufc/g) }\end{array}$ & & \multicolumn{3}{c}{$\begin{array}{c}\text { Productions fruitières } \\
\text { (ufc/g) }\end{array}$} \\
& $A$ & $B$ & $C$ & $D$ & $E$ \\
\hline & & & & & \\
$\mathrm{J}+1$ & $7,3.10^{5}$ & $9,7.10^{5}$ & $2,4.10^{4}$ & $1,9.10^{6}$ & $1,1.10^{6}$ \\
$\mathrm{~J}+8$ & $7,8.10^{8}$ & $1,7.10^{8}$ & $3,7.10^{8}$ & $1,7.10^{9}$ & $2,6.10^{9}$ \\
$\mathrm{~J}+15$ & $6,0.10^{8}$ & $4,0.10^{7}$ & $2,4.10^{8}$ & $1,7.10^{9}$ & $4,0.10^{9}$ \\
$\mathrm{~J}+36$ & $1,3.10^{8}$ & $2,0.10^{7}$ & $3,0.10^{7}$ & $4,0.10^{8}$ & $2,8.10^{9}$ \\
& & & & & \\
\hline
\end{tabular}

\section{DISCUSSION}

Geotrichum candidum est le seul champignon filamenteux du reblochon. Présent dès les stades précoces de toutes les fabrications, il est responsable de la fine "fleur" blanche en surface des reblochons commercialisés. Cette moisissure fréquemment isolée des laits crus (résultats personnels non publiés), forte consommatrice de lactates, est un des agents réputés de la désacidification des pâtes fromagères. II est considéré comme caractéristique des pâtes pressées tel que le Saint Nectaire (Guégen et Lenoir, 1975; Choisy et al, 1987; Marcellino et Benson, 1992). II est aussi présent dans de nombreux fromages à pâte molle comme le Limburger, le Romadour (Neve et Teuber, 1989) et dans les fromages à pâte molle et croûte fleurie tels que le Camembert (Rousseau, 1984). Mais il est rare que Geotrichum candidum soit le seul champignon filamenteux d'une croûte fromagère. Dans le Saint Nectaire, il est en effet associé à du Mucor, du Trichothecium et du Cylindrocarpon et dans la plupart des fromages à croûte fleurie, il est associé à Penicillium camemberti. Dans le cas du reblochon, les rares autres moisissures rencontrées telles que Mucor ou Penicillium ne sont que des contaminants accidentels.
L'évolution quantitative des populations fongiques, levures et moisissures, est la même pour toutes les fabrications. Sur le reblochon, la croissance du Geotrichum et la multiplication des levures se font essentiellement entre $\mathrm{J}+1$ et $\mathrm{J}+8$.

L'importance de la population finale du Geotrichum est indépendante de la technologie mise en œuvre. En particulier, les "frottages" des reblochons fruitiers avec des suspensions de Geotrichum n'entraînent pas une croissance plus rapide de cette moisissure ni une population finale plus importante que celle des reblochons fermiers "non frottés". Vraisemblablement, les populations de $G$ candidum apportées lors des soins sont négligeables comparées aux populations déjà installées sur les fromages.

En ce qui concerne les levures, il faut souligner que la méthodologie utilisée ne permet d'isoler des fromages que les espèces dominantes. Parmi les 13 espèces isolées, 3 , Candida famata, $C$ zeylanoides et $C$ vini sont remarquables par leur fréquence élevée (125 isolats/175) et leur distribution dans les différentes fabrications.

Candida famata, levure du lait cru (Engel, 1986a) est la seule espèce retrouvée sur tous les reblochons. C'est typiquement une 
levure du fromage puisqu'elle est signalée sur le roquefort (Devoyod et Sponem, 1970), le Saint Nectaire (Dale et Guillot, 1971; Vergeade, 1975), le camembert (Schmidt et Lenoir, 1978, 1980), le cabrales (Nunez et al, 1981) et sur de nombreux fromages de chèvre (Nahabieh et Schmidt, 1990). C'est aussi une levure dominante des saumures (Seiler et Busse, 1990).

Candida zeylanoides signalée jusqu'alors uniquement dans les fromages blancs (Engel, 1986b) est très bien implantée sur les reblochons fruitiers. Cette espèce non isolée des productions fermières correspond à $58,7 \%$ des isolats des fabrications fruitières.

Candida vini, absente des productions fruitières, est en revanche l'espèce la plus abondante $(36,4 \%$ des isolats) des reblochons fermiers. A notre connaissance, cette espèce n'a jusqu'alors pas été signalée dans d'autres fromages au-delà des stades précoces.

\section{CONCLUSION}

Comme pour beaucoup de fromages (Devoyod, 1990), c'est essentiellement au cours de la première semaine d'affinage que s'installent et se développent les populations fongiques du reblochon. Mais le reblochon est relativement original par la variété des levures de sa croûte : en effet, 13 espèces différentes et dominantes, toutes connues dans des produits laitiers, ont été isolées lors de l'affinage.

En ce qui concerne les champignons filamenteux, le reblochon est encore original puisque Geotrichum candidum est la seule et unique moisissure de la croûte.

Par ailleurs, ce travail montre que si les niveaux des populations fongiques de surface sont à peu près les mêmes aux différentes étapes de l'affinage pour les produits fermiers et les produits fruitiers, en revanche, le reblochon fermier est nettement plus riche en espèces de levures dominantes que le reblochon fruitier. Ce résultat est un argument en faveur de la réalisation d'un conservatoire des souches fongiques fermières du reblochon, afin d'en préserver les caractéristiques microbiologiques.

\section{RÉFÉRENCES}

Bamett JA, Payne RW, Yarrow D (1990a) Yeasts: characteristics and identification. Cambridge University Press, Cambridge

Barnett JA, Payne RW, Yarrow D (1990b) Yeast identification program. Cambridge University Press, Cambridge

Berthier J, Michel A, Valla G, Bärtschi C (1991) Accidents Mucor en fromagerie : facteurs de risque et prévention. Affiche et article du Colloque Société française microbiologie, Paris, mars 1991 : Les micro-organismes contaminants dans les industries agro-alimentaires

Chamba JF, Perron MO (1991) La microflore de surface du reblochon de Savoie. Rapport ITG $36 \mathrm{p}$

Choisy C, Guéguen M, Lenoir J, Schmidt JL, Tourneur C (1987) Les phénomènes microbiens. In: Le Fromage (Eck A, ed). Tec et Doc Lavoisier, Paris, 259-290

Dale G, Guillot J (1971) Contribution à l'étude de la microflore du fromage de Saint Nectaire et de la physiologie de Geotrichum candidum Link. CR Soc Biol 165, 309-316

Deak T (1993) Simplified techniques for identifying foodborne yeasts. Int J Food Microbiol 19, 15-26

Deak T, Beuchat LR (1988) Evaluation of simplified and commercial systems for identification of foodborne yeasts. Int J Food Microbiol 7 , 135-145

De Hoog GS, Smith MTH, Guého E (1986) A revision of the genus Geotrichum and its teleomorphs. Stud Mycol 29, Centraalbureau voor Schimmelcultures Baarn and Delft, 1-131

Devoyod JJ (1990) Yeast in cheese-making. In: Yeast technology (Spencer J, Spencer D, eds). Springer-Verlag Berlin Heidelberg, 228240 
Devoyod JJ, Sponem D (1970) La flore microbienne du fromage de roquefort. VI. Les levures. Lait 50, 524-543

Domsch KH, Gams W, Anderson TH (1980) Compendium of soil fungi. Academic Press Ltd, London

Ellis MB (1971) Dematiaceous Hyphomycetes. Commonwealth Agric Bur, Aberystwyth

Ellis MB (1976) More dematiaceous Hyphomycetes. Commonwealth Agric Bur, Aberystwyth

Engel G (1986a) Hefen in Silagen und Rohmlich. Milchwissenschaft 41, 633-636

Engel G (1986b) Vorkommen von Hefen in Frischkäse - organoleptische Beeinflussung. Milchwissenschaft 41, 692-694

Fowell RR (1952) Sodium acetate agar as a sporulation medium for yeast. Nature 170, 578

Guéguen M, Lenoir J (1975) Aptitude de l'espèce Geotrichum candidum à la production d'enzymes protéolytiques. Lait 55, 145-152

Heard G, Fleet G (1990) A convenient microtitre tray procedure for yeast identification. J Appl Bacteriol 68, 447-451

Ison RW (1987) A revised method for the application of API $50 \mathrm{CH}$ carbohydrate kits to yeasts. Lett App/ Microbiol 4, 9-11

Marcellino N, Benson D (1992) Scanning electron and light microscopic study of microbial succession on Bethlehem Saint Nectaire cheese. Appl Environ Microbiol 58, 3448-3454

Nahabieh F, Schmidt JL (1990) Contribution à l'étude de la flore levure de quelques grands types de fromages de chèvre. Lait 70, 325343

Neve H, Teuber M (1989) Scanning electron microscopy of the surface microflora of ripened soft cheeses. Kiel Michwirtsch Forschungsber 41, 3-13

Nunez M, Medina M, Gaya P, Diaz-Amado C (1981) Les levures et les moisissures dans le fromage bleu de Cabrales. Lait 61, 62-79

Rohm H, Lechner F, Lehner M (1990) Evaluation of the API ATB $32 C$ system for the rapid identification of foodborne yeasts. Int J Food Microbiol 11, 215-224

Rousseau M (1984) Study of the surface flora of traditional Camembert cheese by scanning electron microscopy. Milchwissenschaft 39 , 129-135

Schmidt JL, Lenoir J (1978) Contribution à l'étude de la flore levure du fromage de Camembert. I. Son évolution au cours de la maturation. Lait 58, 355-370

Schmidt JL, Lenoir J (1980) Contribution à l'étude de la flore levure du fromage de Camembert. II. Lait $60,272-282$

Schmidt JL, Daudin JJ (1983) Étude de l'homogénéité d'une population de levures isolées à partir de fromages de Camembert. Ann Microbiol Inst Pasteur 134A, 399-409

Seiler H (1991) Some additional physiological characteristics for the identification of foodborne yeasts. Neth Milk Dairy J 45, 253-258

Seiler H, Busse M (1990) The yeasts of cheese brines. Int J Food Microbiol 11, 289-304

Sutton BC (1980) The Coelomycetes. Commonwealth Agric Bur, Glasgow

Vergeade J (1975) Etude des levures du fromage de Saint Nectaire. Thèse de Doctorat $3^{e}$ cycle, Univ Clermont-Ferrand

Wickerham LJ (1951) Taxonomy of yeasts. Tech Bull US Dep Agric 1029, 1-56 